\title{
DETERMINACIÓN DE TASAS DE GENERACIÓN DE VIAJES PARA COLEGIOS PÚBLICOS UBICADOS EN LA CIUDAD DE GUAYAQUIL, ECUADOR
}

\author{
DETERMINATION OF TRAVEL GENERATION RATES FOR PUBLIC SCHOOLS \\ LOCATED IN THE CITY OF GUAYAQUIL, ECUADOR
}

\section{FEDERICO G. VON BUCHWALD DE JANON', ANDRÉS BERREZUETA VÉLEZ², CAROLINA GÓMEZ MALDONADO ${ }^{3}$}

\author{
1 Universidad Católica de Santiago de Guayaqui l. fritzvb25@icloud.com \\ 2 Universidad Católica de Santiago de Guayaqui l. andres.berrezueta@cu.ucsg.edu.ec \\ 3 Departamento Técnico de Consultora y Auditora Consuladi Cía. Ltda., Ecuador. carolinagomezmaldonado@hotmail.com
}

RESUMEN

En la actualidad, cuando se ha necesitado conocer localmente los viajes que genera un determinado tipo de instalación se ha usado como parámetro el manual del ITE "Trip Generation", el mismo que presenta datos de generación de viajes de distintos usos de suelos en Estados Unidos, siendo estos datos no aplicables en su totalidad en nuestro entorno ya que presentan una media estadística de lugares con realidades socioeconómicas distintas a las de la ciudad de Guayaquil. Esta investigación está basada en medir los volúmenes de viajes de los polos generadores de viajes: colegios públicos de Guayaquil, para lo cual se realizaron conteos manuales en las puertas de ingreso de los colegios desde las 6:00 hasta las 7:30, horario de ingreso en las instalaciones educativas, logrando así determinar la cantidad de alumnos, profesores y personal administrativo que llegó a la institución educativa y los viajes que estos generaron. Las variables independientes con las que se trabajó fueron número de estudiantes y número de empleados. Los resultados finalmente obtenidos, demuestran que las tasas de generación de viajes locales son inferiores a las tasas de generación de Estados Unidos en colegios públicos, tanto para la variable independiente estudiantes como empleados. En la relación entre el número de viajes y el número de estudiantes de los colegios públicos, se obtuvo que en el caso de menor número de estudiantes (600) los coeficientes de correlación es de 0,16 y en el caso de mayor número de estudiantes (2500) el valor es de 0,32; al comparar estos resultados con los obtenidos del ITE, para el caso de mayor número de estudiantes el coeficiente de correlación es 1,4 veces mayor, y en el caso de menor número de alumnos la relación es 2,6 veces mayor al que se genera realmente. Para el caso de número de empleados para el menor número (50), la relación viajes/número de empleados resulto 0,56 y para el mayor número de empleados (250), 3,88. Comparando con los resultados del ITE, las relaciones de los viajes del ITE son 7,87 para el mayor número de empleados y 1,11 para el menor número de empleados.

PALABRAS CLAVE: polos generadores de viajes, generación de viajes, colegios públicos, estudiantes, empleados, coeficientes de correlación. Nowadays, the Trip Generation Manual from ITE (USA) has been used as a parameter for the land use trips. These data is not fully applicable in our city since they present a statistical average of places with different socioeconomic realities from those of the city of Guayaquil. This research is based on measuring the travel volumes of the travel generator pole: public schools in Guayaquil, for which manual counts were made at the entrance doors of the schools from 6:00 am to 7:30 am (schedule entrance in educational facilities), to determine the number of students, teachers and administrative staff who arrived at the educational institution and the trips that these generate. The independent variables used were number of students and number of employees. The results show that local travel generation rates are lower than the United States generation rates in public schools, for both of the independent variables: students and employees. In the relationship between the number of trips and the number of students in public schools, it was obtained that in the case of a smaller number of students (600) the correlation coefficients are 0,16 and in the case of a larger number of students (2500) the value is 0,32 . When comparing these results with those obtained from the ITE, for the case of a greater number of students the correlation coefficient is 1,4 times higher, and in the case of a smaller number of students the ratio is 2,6 times greater than the one really generated.

For the case of number of employees for the smallest number (50), the number of trips / employee ratio was 0,56 and for the largest number of employees (250), 3,88. Comparing with the ITE results, the ITE travel ratios are 7,87 for the largest number of employees and 1,11 for the smallest number of employees.

KEYWORDS: Travel generators poles, trip generation, public schools, students, employees, correlation coefficients. 


\section{INTRODUCCIÓN}

La planificación del transporte en toda ciudad está comprendida dentro de un proceso denominado modelo de transporte de cuatro etapas, las mismas que son: Generación de viajes, distribución de viajes, selección modal y asignación modal. (Rosas Meza, 2012). De las fases mencionadas, la generación de viajes busca, en base a las características socio-económicas de la población, estimar la cantidad de viajes que entran y salen de una zona, además tiene como objetivo reducir los impactos producidos mayormente por alteraciones en la zonificación.

Dada la necesidad de planificar eficientementeel desarrollourbanoy vial delas ciudades, en Estados Unidos el Institute of Transportation Engineers (ITE) ha desarrollado el manual "Trip Generation”, el mismo que reúne extensos datos de generación de viajes obtenidos mediante conteos y encuestas a todo lo largo de dicho país. Este manual es una poderosa herramienta para municipios y desarrolladores de proyectos, ya que permite estimar la cantidad de viajes vehiculares que generará un determinado tipo de instalación, según su uso de suelo.

En Guayaquil se busca desarrollar tablas propias a fin de obtener el dato de viajes que se producen en los colegios públicos, por lo que se hicieron conteos en las puertas de ingreso para obtener las correlaciones entre los viajes y las variables independientes, alumnos y empleados. Adicional a esto, se obtuvo el cálculo de la tasa promedio de viajes, la desviación estándar, la ecuación de regresión y finalmente el gráfico similar al del ITE que relaciona la variable independiente estudiada con la cantidad de viajes para una unidad de tiempo determinada.

ANTECEDENTES

La ciudad de Guayaquil, ubicada el delta del golfo del mismo nombre, que desemboca en el Océano Pacífico, es la ciudad de mayor población de la República del Ecuador, en la actualidad cuenta con una población estimada de 2'350.915 habitantes (INEC, 2010) y con ciudades cercanas a menos de 40 kilómetros que totalizan 2'840.508 habitantes (incluyendo Nobol, Samborondón, Durán, Milagro y Daule). Considerando su condición de ciudad grande, la misma acarrea los típicos desafíos de movilidad que presentan otras ciudades con demografía y condiciones socioeconómicas similares. Dentro de estos desafíos, principalmente es de destacar el crecimiento del parque automotor, que a su vez se traduce en problemas de congestionamiento vial.
El Institute of Transportation Engineers (ITE) de Estados Unidos, desarrolló el manual de Trip Generation, que es una herramienta bibliográfica para estimar la cantidad de viajes que genera un polo generador de viajes en función de una variable independiente y permite una correcta planificación de las vías de acceso y demás infraestructura necesaria para un correcto desarrollo urbano. Sin embargo, el uso de datos del ITE para proyectos en la ciudad de Guayaquil podría no ajustarse a la realidad, ya que las distintas condiciones culturales y socioeconómicas generan distintos tipos de demanda de viajes. Entre algunas de estas distintas características, se encuentra que el $56 \%$ de los desplazamientos urbanos en Guayaquil se realizan en transporte público (considerando todas las modalidades de transporte), y que la tasa de motorización es aproximadamente del $12 \%$ mientras que en Estados Unidos la misma tasa pueden llegar a un 80\% (von Buchwald de Janón, 2014).

Pese a la gran magnitud poblacional de Guayaquil, actualmente esta ciudad no tiene datos de generación de viajes reales según los diversos tipos de uso de suelo, además no cuenta con un instituto o centro de estudio que se dedique a la investigación de generación de viajes.

\section{JUSTIFICACIÓN}

Dado que en la ciudad no se tiene un estudio de base para determinar cuántos viajes generan los colegios públicos y que se conoce que tienen una alta concentración del 59\% del alumnado del total del sistema educativo de Guayaquil (MINISTERIO DE EDUCACION, 2012). Estos datos permitirán la planificación y el desarrollo de infraestructura vial y de transporte que permita contrarrestar los posibles impactos negativos que ocasionan en la vialidad.

OBJETIVO

Determinar las tasas de generación de viajes en distintos colegios públicos de la ciudad de Guayaquil.

\section{MATERIAL Y MÉTODO}

Se usó como guía para este estudio el "Trip Generation Manual 9th Edition" Volumen 1 (User's guide and Handbook) y Volumen 3 (Data), el cual provee guías paso a paso para la conducción de estudios de generación de viajes.

DESCRIPCIÓN DEL GRÁFICO DE GENERACIÓN DE VIAJES Y REPORTES ESTADÍSTICOS

La gráfica de datos es la parte fundamental del estudio de generación de viajes. Es importante 
señalar que los puntos base representados en las gráficas no son las tasas de generación de viajes, sino que representan el número total viajes observados contra el tamaño de la variable independiente.

Algunas gráficas del Trip Generation, hechas a base de un pequeño número de muestras, suelen contener un aviso que indica que es preferible tener precaución con el uso que se le dará a la gráfica, puesto que al desarrollarla con cinco o menos la convierte en una muestra menos confiable y puede comprometer la base de datos (ITE, 2012).

En la ilustración a continuación se puede observar como el ITE presenta las gráficas en su formato:

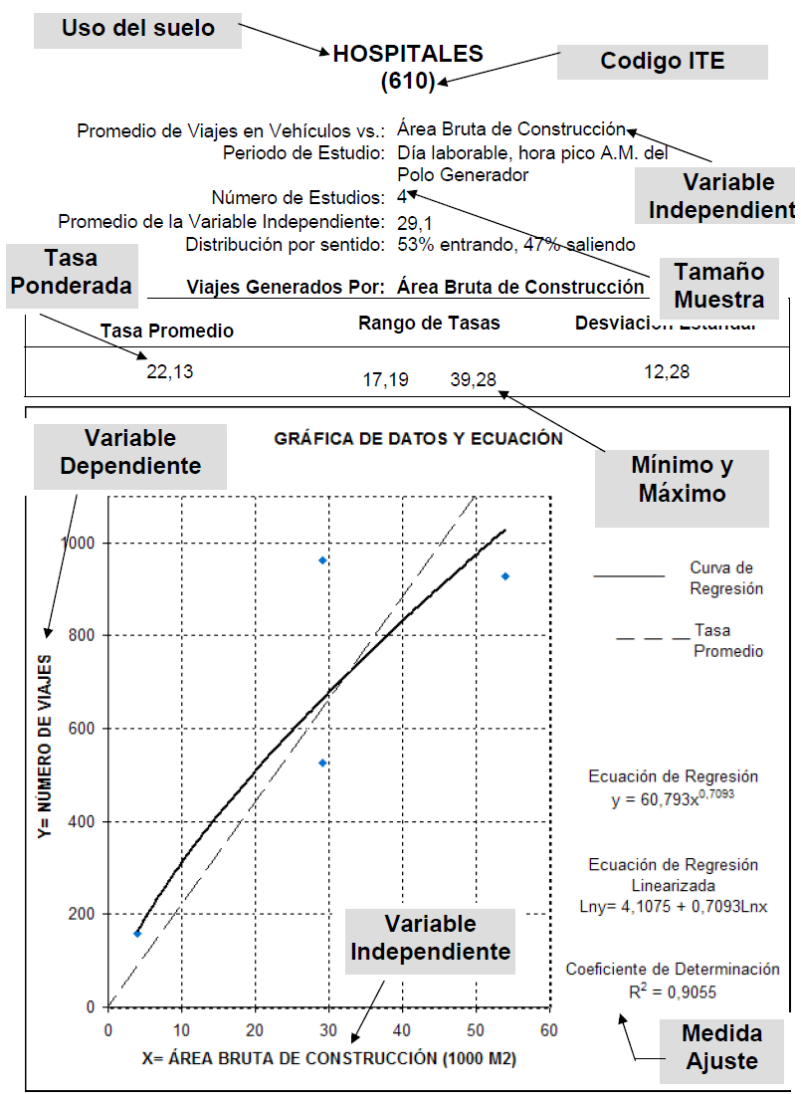

Ilustración 1. Modelo explicativo del contenido de los gráficos de ITE. Fuente: Rosas Meza (2012).

COLEGIOS SELECCIONADOS PARA ESTUDIO:

1. Vicente Rocafuerte

2. 28 de Mayo

3. Aguirre Abad

4. Unidad Educativa Réplica Aguirre Abad

5. Simón Bolívar

6. Dolores Sucre

7. Augusto Mendoza Moreira

8. Unidad Educativa Ati II Pillahuaso

9. Martha Bucaram de Roldós

10. Unidad Educativa Adolfo H. Simmonds
SELECCIÓN DE LAS VARIABLES INDEPENDIENTES

Las variables independientes seleccionadas se basaron en el manual del ITE, las cuales se describen a continuación:

- Número de estudiantes (principal variable independiente).

- Empleados

Además de las variables independientes usadas, el Trip Generation incluye análisis de viajes con la variable "área bruta de construcción" que está en unidades de mil pies cúbicos, sin embargo, la misma no ha sido considerada para este estudio dadas las dificultades en poder conseguir datos confiables.

Pese a que la mayoría de los colegios seleccionados ofertan distintos horarios de educación, se trabajó únicamente con los datos de alumnos de las secciones matutinas de cada uno de los colegios.

\section{METODOLOGÍA PARA EL LEVANTAMIENTO DE LA INFORMACIÓN}

Se realizaron conteos manuales en las puertas de ingreso de los colegios durante dos días laborables en el horario de ingreso a las instituciones educativas, ya que es muy marcada la tendencia de horas pico en este tipo de uso de suelo. Los conteos empezaron a las $6 \mathrm{H} 00$ y se llevaron a cabo hasta las 7H30, separando los viajes en períodos de quince minutos. El análisis se enfocó en el ingreso y evitó considerar los viajes generados durante el horario de salida, en el que las actividades extracurriculares y los horarios de colegios vespertinos causan fluctuaciones según el día analizado.

Como parte del trabajo de investigación, se consideraron varios tipos de vehículos, siendo estos: motos, autos, buses, furgonetas o busetas y camiones.

Para determinar la representatividad de los días en los que se hicieron los conteos, ya que no se podía hacer un conteo durante todo el año porque se tenía un límite de tiempo para que el investigador presente los resultados, se tomaron los datos del número de pasajeros de las 3 Troncales del sistema de transporte público BRT de Guayaquil, Metrovia, en cada uno de los días en los que se realizaron los conteos y se los comparó con el promedio de pasajeros de la Metrovia en un día laborable en el año de estudio durante los meses de mayo a enero en los que los colegios tienen clases, el cual resultó ser de 453.065 pasajeros y, se obtuvo que los días 


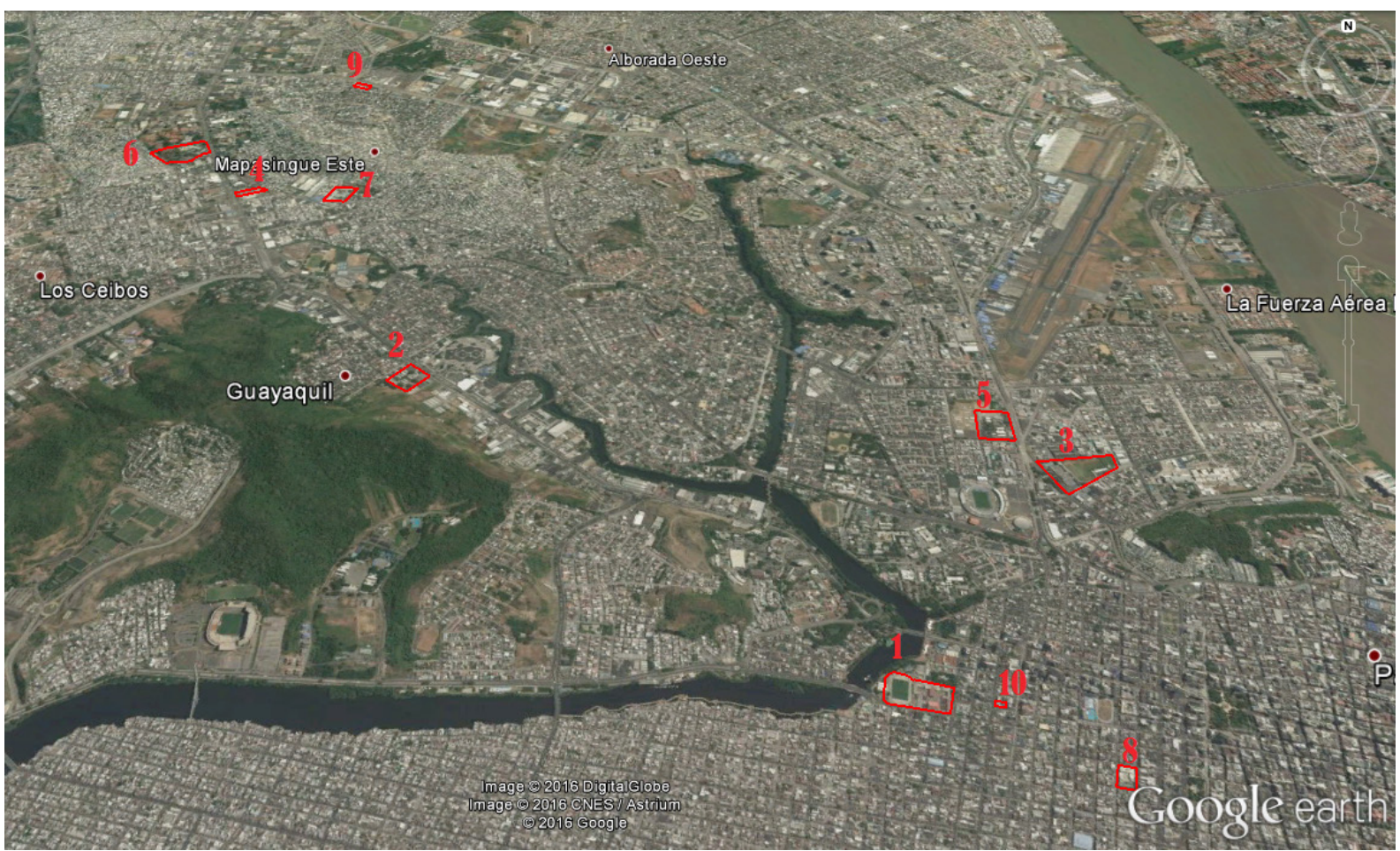

Figura 2. Ubicación de colegios estudiados

Fuente: Elaboración Propia - Google Earth Pro.

de conteos representan un 108\% (promedio ponderado de los días de conteos) del día promedio. Con esta condición se han realizado ajustes en los volúmenes de viajes a un $8 \%$ menos.

\section{RESULTADOS Y DISCUSIÓN OBTENCIÓN DE TASAS DE GENERACIÓN DE VIAJES}

Las tasas obtenidas de generación de viajes de los colegios públicos han sido determinadas a partir de la recopilación de la información de este estudio. Estas tasas son presentadas con los resultados de tasa promedio ponderadas, rango de tasas y desviación estándar. Además, mediante el análisis de regresión, se elige la ecuación de la gráfica que permita relacionar la variable dependiente con las variables independientes correspondientes.

La tabla a continuación muestra las tasas de generación de viajes de todos los colegios estudiados por cada variable independiente y durante su periodo pico A.M. de ingreso a los colegios.

TABLA 1. TASAS DE GENERACIÓN DE VIAJES-HORA PICO AM

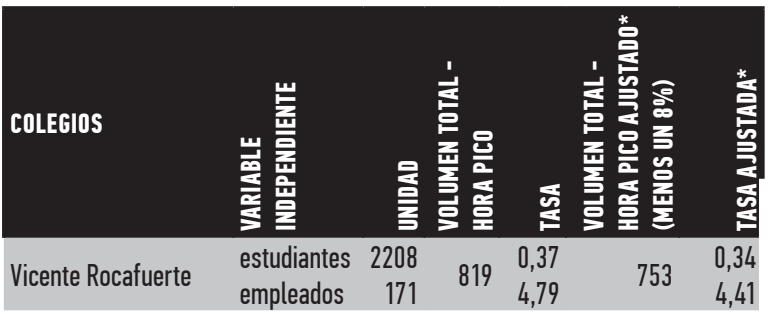

\begin{tabular}{|c|c|c|c|c|c|c|}
\hline COLEGIOS & 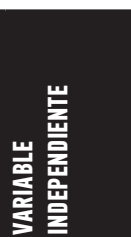 & 言 & 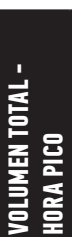 & 畄 & 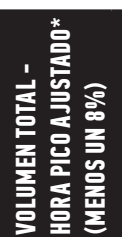 & 䓂 \\
\hline \multirow{2}{*}{28 de Mayo } & estudiantes & 2650 & \multirow{2}{*}{930} & 0,35 & \multirow{2}{*}{855} & 0,32 \\
\hline & empleados & 216 & & 4,30 & & 3,96 \\
\hline \multirow{2}{*}{ Aguirre Abad } & estudiantes & 1456 & \multirow{2}{*}{501} & 0,34 & \multirow{2}{*}{461} & 0,32 \\
\hline & empleados & 141 & & 3,55 & & 3,27 \\
\hline \multirow{2}{*}{$\begin{array}{l}\text { Unidad Educativa } \\
\text { Réplica Aguirre Abad }\end{array}$} & estudiantes & 885 & \multirow{2}{*}{158} & 0,18 & \multirow{2}{*}{145} & 0,16 \\
\hline & empleados & 85 & & 1,86 & & 1,71 \\
\hline \multirow{2}{*}{ Simón Bolívar } & estudiantes & 1098 & \multirow{2}{*}{297} & 0,27 & \multirow{2}{*}{273} & 0,25 \\
\hline & empleados & 132 & & 2,25 & & 2,07 \\
\hline \multirow{2}{*}{ Dolores Sucre } & estudiantes & 1940 & \multirow{2}{*}{427} & 0,22 & \multirow{2}{*}{393} & 0,20 \\
\hline & empleados & 150 & & 2,85 & & 2,62 \\
\hline \multirow{2}{*}{$\begin{array}{l}\text { Augusto Mendoza } \\
\text { Moreira }\end{array}$} & estudiantes & 1225 & \multirow{2}{*}{220} & 0,18 & \multirow{2}{*}{202} & 0,17 \\
\hline & empleados & 98 & & 2,24 & & 2,07 \\
\hline \multirow{2}{*}{$\begin{array}{l}\text { Unidad Educativa Ati II } \\
\text { Pillahuaso }\end{array}$} & estudiantes & 1400 & \multirow{2}{*}{676} & 0,48 & \multirow{2}{*}{622} & 0,44 \\
\hline & empleados & 110 & & 6,15 & & 5,65 \\
\hline \multirow{2}{*}{$\begin{array}{l}\text { Martha Bucaram de } \\
\text { Roldós }\end{array}$} & estudiantes & 900 & \multirow{2}{*}{222} & 0,25 & \multirow{2}{*}{204} & 0,23 \\
\hline & empleados & 59 & & 3,75 & & 3,45 \\
\hline \multirow{2}{*}{$\begin{array}{l}\text { Unidad Educativa } \\
\text { Adolfo H. Simmonds }\end{array}$} & estudiantes & 612 & \multirow{2}{*}{135} & 0,22 & \multirow{2}{*}{124} & 0,20 \\
\hline & empleados & 60 & & 2,24 & & 2,06 \\
\hline
\end{tabular}

Nota: Generación de viajes ajustados a las circunstancias de colegios públicos de la ciudad de Guayaquil, Ing. Andrés Berrezueta (2016).

A continuación, se presenta los cálculos de las tasas promedios ponderadas, rango de tasas y desviación estándar de cada una de las variables independientes. 
TABLA 2. TASAS DE GENERACIÓN DE VIAJES

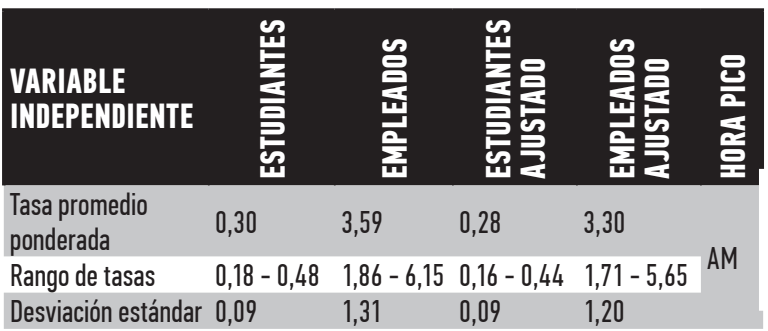

Nota: Generación de viajes ajustados a las circunstancias de colegios públicos de la ciudad de Guayaquil, Ing. Andrés Berrezueta (2016).

ANÁLISIS DE REGRESIÓN

Las ecuaciones de regresión se obtuvieron de las gráficas finales. Una vez que se dispuso de los datos de variables dependientes e independientes, estas se graficaron como puntos dispersos con un valor para el eje X y para el eje $\mathrm{Y}$, obteniendo tantos puntos como colegios se han estudiado (10 colegios).

Una vez graficados los datos, por medio de las herramientas gráficas de la hoja de cálculo de Microsoft Excel, se obtuvieron las ecuaciones lineal y logarítmica para la línea de tendencia que mejor se ajusta a los datos graficados, se obtuvo además el coeficiente de correlación $\mathrm{R}^{2}$, y se escogió la curva con el coeficiente más alto que se traduce en un mejor ajuste, de los modelos matemáticos utilizados para determinar la ecuación de regresión, el modelo lineal fue e] que mejor se ajustó a los puntos graficados ofreciendo el mejor coeficiente de determinación $\left(\mathrm{R}^{2}\right)$ para la variable independiente.

Las tasas de generación de viajes obtenidas durante este estudio, con valores altos de coeficientes de correlación mayores a 0,5. Las tablas 3 y 4 garantizan de manera efectiva que los gráficos finales contienen información útil y bastante aproximada para predecir el comportamientc de la demanda de viajes en los colegios públicos de la ciudad de Guayaquil. Estas tasas podrán ser usadas como herramienta tanto para pla- nificadores de futuros proyectos escolares como cualquier persona que se halle en la necesidad de conocer la demanda de viajes de colegios públicos en función del número de estudiantes o del número de empleados de la institución.

\section{GRÁFICAS DE GENERACIÓN DE VIAJES}

Las gráficas, tanto la del ITE, como la de datos locales, comparten paralelismo en toda su extensión, con apenas un desfase en la cantidad de viajes, que es menor en la ciudad de Guayaquil. Esto es aplicable tanto para el caso de la variable alumnos como de la variable empleados. En las siguientes tablas, se presentan además las curvas ajustadas al análisis de los pasajeros de la Metrovía, el que en los días de conteo resultó ser un $8 \%$ más que el día promedio, por lo que se ajustaron los datos restándole el $8 \%$ a los puntos que se tenían.

COLEGIOS PÚBLICOS

USO DE SUELO: 530 (CODIGO ITE)

\begin{tabular}{c}
\hline PROMEDIO DE VIAJES CONTRA: ESTUDIANTES \\
EN DIA: ENTRE SEMANA \\
HORA PICO MANNANA (A.M.) \\
Número de colegios estudiados: 10 \\
Número promedio de estudiantes: 1437 \\
GENERACION DE VIAJES POR ESTUDIANTE: \\
TASA PROMEDIO \\
RANGO DE TASAS \\
0,28
\end{tabular}

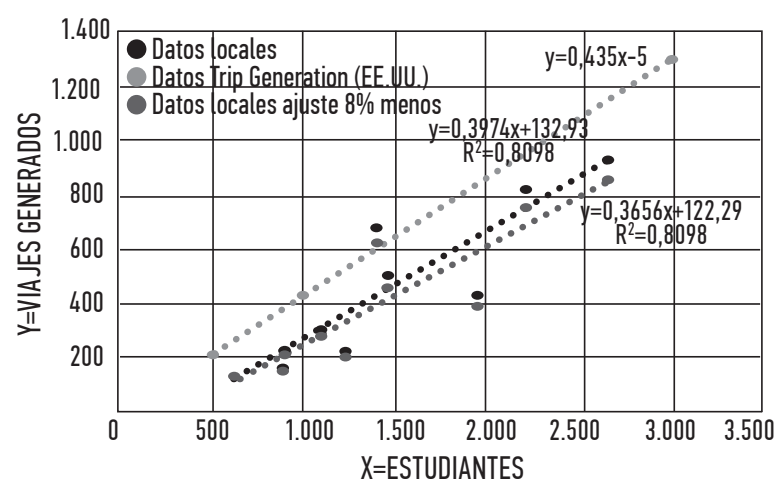

Figura 3. Generación de Viajes para el número de estudiantes, hora pico AM Fuente: Berrezueta (2016)

TABLA 3. ECUACIÓN DE REGRESIÓN Y COEFICIENTE DE CORRELACIÓN PARA LA VARIABLE INDEPENDIENTE "NÚMERO DE ESTUDIANTES" OBTENIDA DE GRAFICA PERIODO AM

\begin{tabular}{|c|c|c|c|c|c|c|}
\hline $\begin{array}{l}\text { PERÍODO DE } \\
\text { ESTUDIO }\end{array}$ & $\begin{array}{l}\text { VARIABLE } \\
\text { INDEPENDIENTE }\end{array}$ & $\begin{array}{l}\text { TIPO DE CURVA } \\
\text { AJUSTADA }\end{array}$ & $\begin{array}{l}\text { ECUACIONES DE } \\
\text { REGRESIÓN }\end{array}$ & $\begin{array}{l}\text { COEFICIENTES DE } \\
\text { CORRELACIÓN }\end{array}$ & $\begin{array}{l}\text { ECUACIONES DE REGRESIÓN } \\
\text { CURVA AJUSTADA (MENOS 8\%) }\end{array}$ & $\begin{array}{l}\text { COEFICIENTES DE } \\
\text { CORRELACIÓN CURVA } \\
\text { AJUSTADA (MENOS 8\%) }\end{array}$ \\
\hline lora pico AM & $\begin{array}{l}\text { Número de } \\
\text { Estudiantes }\end{array}$ & Lineal & $\begin{array}{l}y=0,3974 x-132,93 \\
y=554,53 \ln (x)-3543\end{array}$ & $\begin{array}{l}R^{2}=0,8731 \\
R^{2}=0,7762\end{array}$ & $\begin{array}{l}y=0,3656 x-122.29 \\
y=510.17 \ln (x)-3259.6\end{array}$ & $\begin{array}{l}R^{2}=0,8098 \\
R^{2}=0,7762\end{array}$ \\
\hline
\end{tabular}

Nota: Generación de viajes ajustados a las circunstancias de colegios públicos de la ciudad de Guayaquil, Ing. Andrés Berrezueta (2016).

TABLA 4. ECUACIÓN DE REGRESIÓN Y COEFICIENTE DE CORRELACIÓN PARA LA VARIABLE INDEPENDIENTE "NÚMERO DE EMPLEADOS" OBTENIDA DE GRAFICA PERIODO AM

$\begin{array}{lllllll}\begin{array}{l}\text { PERÍODO DE } \\ \text { ESTUDIO }\end{array} & \begin{array}{l}\text { VARIABLE } \\ \text { INDEPENDIENTE }\end{array} & \begin{array}{l}\text { TIPO DE CURVA } \\ \text { AJUSTADA }\end{array} & \begin{array}{l}\text { ECUACIONES DE } \\ \text { REGRESIÓN }\end{array} & \begin{array}{l}\text { COEFICIENTES DE ECUACIONES DE REGRESIÓN } \\ \text { CORRELACIÓN }\end{array} & \begin{array}{l}\text { COEFICIENTES DE } \\ \text { CURVA AJUSTADA (MENOS 8\%) }\end{array} \\ \text { CORRELACIÓN CURVA } \\ \text { AJUSTADA (MENOS 8\%) }\end{array}$

Nota: Generación de viajes ajustados a las circunstancias de colegios públicos de la ciudad de Guayaquil, Ing. Andrés Berrezueta (2016). 


\begin{tabular}{|c|c|c|}
\hline \multicolumn{3}{|c|}{$\begin{array}{c}\text { COLEGIOS PÚBLICOS } \\
\text { USO DE SUELO: } 530 \text { (CODIGO ITE) }\end{array}$} \\
\hline \multicolumn{3}{|c|}{ PROMEDIO DE VIAJES CONTRA: EMPLEADOS } \\
\hline \multicolumn{3}{|c|}{ EN DIA: ENTRE SEMANA } \\
\hline \multicolumn{3}{|c|}{ HORA PICO MAÑANA (A.M.) } \\
\hline \multicolumn{3}{|c|}{ Número de colegios estudiados: 10} \\
\hline \multicolumn{3}{|c|}{ Número promedio de estudiantes: 122} \\
\hline \multicolumn{3}{|c|}{ GENERACION DE VIAJES POR ESTUDIANTE: } \\
\hline TASA PROMEDIO & RANGO DE TASAS & DESVIACIÓN ESTÁNDAR \\
\hline 3,30 & $1,71-5,65$ & 1,20 \\
\hline
\end{tabular}

Figura 4. Generación de Viajes para el número de empleados, hora pico AM. Fuente: Berrezueta (2016).

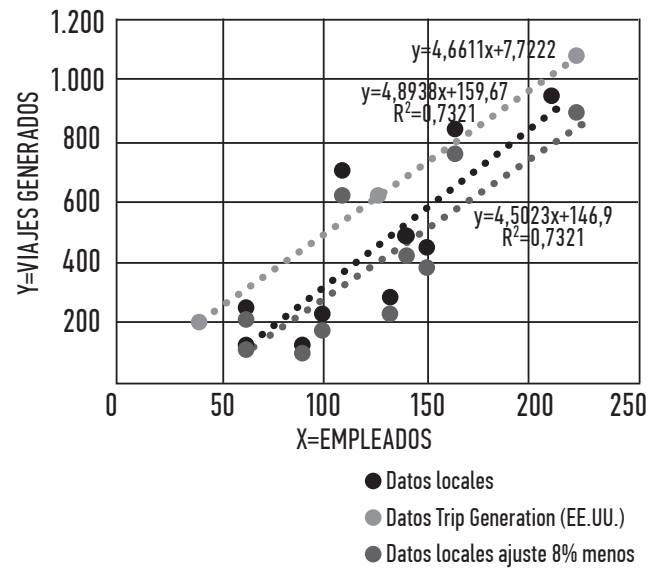

CONCLUSIONES

- En colegios muy numerosos tanto en alumnos como empleados, se puede esperar un comportamiento de demanda de viajes similar a los obtenidos en los estudios de Estados Unidos, no siendo el mismo caso en colegios públicos con pocos alumnos.

- Haciendo una relación entre el número de viajes y el número de estudiantes de los colegios públicos, se obtuvo que en el caso de menor número de estudiantes (600) la relación es de 0,18 $\mathrm{y}$ en el caso de mayor número de estudiantes (2500) el valor es de 0,35 , realizando el ajuste de acuerdo al volumen de pasajeros de la Metrovia, los valores resultan 0,16 y 0,32 respectivamente. Para el caso de número de empleados para el menor número (50), la relación viajes/número de empleados resulto 0,61 y para el mayor número de empleados (250), 4,21 y con el ajuste al volumen de viajes los valores corregidos resultaron 0,56 y 3,88 respectivamente.

- Al comparar estos resultados con los del ITE se obtiene que para el caso de mayor número de estudiantes la relación es de 1,3 veces mayor, y en el caso de menor número de alumnos la relación es 2,4 veces mayor. Realizado el ajuste de los viajes a un $8 \%$ menos, se tuvo que las relaciones cambiaron a 1,4 y 2,6 respectivamente. En el caso de número de empleados las relaciones de los viajes del ITE resultaron ser 7,9 para el mayor número de empleados y 1,1 para el menor número de empleados, y con los viajes ajustados estos valores son 7,87 y 1,11 respectivamente.

- El análisis tanto para la variable dependiente Alumnos como para el Número de empleados, tiene similitud en pendiente con las curvas de ITE, aunque obteniendo en ambos casos menores valores que los de Estados Unidos.

\section{REFERENCIAS BIBLIOGRÁFICAS}

Berrezueta , A. (2016). Generación de viajes ajustados a las circunstancias de colegios públicos de la ciudad de Guayaquil. Guayaquil: Universidad Católica Santiago de Guayaquil.

Ciafardo, R., Lagos, S., Segovia, M., \& Vallejos, V. H. (s.f.). EL TRANSPORTE COMO UN INDICADOR DE CALIDAD DE VIDA. Recuperado el 16 de Mayo de 2016, de http://www.perfilciutat.net/fitxers/ IVSL_B1.pdf

Ciencia e Ingeniería . (2011). Ciencia e Ingeniería.

Garber N, Hoel L. (2005). Ingeniería de Tráfico y Carreteras. Mexico: Thomson Editores.

INEC. (2010). Resultados Censo de Población . Obtenido de Instituto Nacional de Estadisticas y Censos: http://www.ecuadorencifras.gob.ec/censo-depoblacion-y-vivienda/

Institute of Transportation Engineers, ITE. (2012). TRIP GENERATION MANUAL.

ITE (2004). Trip Generation Handbook 2ND ED.

MINISTERIO DE EDUCACION. (2012). Archivo Maestro de Instituciones Educativas - AMIE.

Molinero, A. R., \& Sánchez, L. I. (1996). Transporte Público: Planeación, Diseño, Operación y Administración. (2da ed.). México.

RED IBEROAMERICANA DE POLOS GENERADORES DE VIAJES, (Red de PGV'S). (s.f.). Conceptos Básicos. Obtenido de http://redpgv.coppe.ufrj. br/index.php/es/conceptos/que-es-um-pgv

Rosas Meza, A. (2012). Estimacion de tasas de Generacion de Viajes para hospitales en el distrito Metropolitano de Caracas.

SENPLADES. (2013). Dirección de Métodos, Análisis e Investigación. Recuperado el 26 de Agosto de 2016, de Proyecciones y Estudios Demográficos - Sistema Nacional de Información: http://app. sni.gob.ec/sni-link/sni/Portal\%20SNI\%202014/ ESTADISTICA/Proyecciones_y_estudios_demograficos/Proyecciones $\% 202010 /$ Proyecciones $\% 20$ de $\% 20$ poblaci $\%$ c $3 \%$ b3n $\% 20$ PARROQUIAL $\% 20$ 2010-2020.xlsx

von Buchwald de Janón, F. (2014). Movilidad Urbana de Guayaquil. Guayaquil: Dirección de Publicaciones UCSG.

Wong Chauvet, D. (2005). Del caos al orden. Cuayaquily su desarrollo urbano actual. 